\title{
Thinking through prior bodies: autonomic uncertainty and interoceptive self-inference
}

\author{
Micah Allen $1,2,3$, Nicolas Legrand, ${ }_{1}$ Camile Correa1, Francesca Fardo1,4 \\ ${ }_{1}$ Center of Functionally Integrative Neuroscience, Aarhus University Hospital, Denmark \\ ${ }_{2}$ Aarhus Institute of Advanced Studies, Aarhus University, Denmark \\ ${ }_{3}$ Cambridge Psychiatry, University of Cambridge, Cambridge, UK \\ ${ }_{4}$ Danish Pain Research Centre, Aarhus University Hospital, Denmark
}

\begin{abstract}
:
The Bayesian Brain Hypothesis, as formalized by the Free Energy Principal, is ascendant in the cognitive sciences. But how does the Bayesian Brain obtain prior beliefs? Veissière and colleagues argue that sociocultural interaction is one important source. We offer a complementary model in which "interoceptive self-inference" guides the estimation of expected uncertainty both in ourselves and in our social conspecifics.
\end{abstract}

Invited BBS Commentary on: Veissière, S. P. L., Constant, A., Ramstead, M. J. D., Friston, K. J., \& Kirmayer, L. J. (2019). Thinking Through Other Minds: A Variational Approach to Cognition and Culture. Behavioral and Brain Sciences, 1-97.

https://doi.org/10.1017/S0140525X19001213

In their impressive synthesis, Veissière and colleagues argue that enactive social interaction is a prime ground for generating higher-order prior beliefs (both implicit and explicit). We share this enthusiasm for social-cultural patterning of priors, and also their comprehensive embrasure of the enactive and embodied turn within the larger predictive processing movement (Allen \& Friston, 2018; Barrett \& Simmons, 2015; Gallagher \& Allen, 2018; M. J. D. Ramstead, Kirchhoff, Constant, \& Friston, 2019 ; M. J. Ramstead, Kirchhoff, \& Friston, 2019; Seth, 2013). As they elegantly argue, ontogenetic development provides a wealth of knowledge about how to behave in a given context. It follows that this 'duet for one' of mutual prediction not only constrains how we engage with others, but also our own self-inference (K. Friston \& Frith, 2015; K. J. Friston \& Frith, 2015). As such the proposal that much of our "repertoire of prior beliefs" emerges from socio-cultural interaction and enactive, embodied engagement is both feasible and exciting.

However, we disagree that "information from and about other people's expectations constitutes the primary domain" from which prior beliefs about "statistical regularities" (i.e., expected precision) arise. While socio-cultural sources certainly contribute, we highlight the predominance of more than 1 million years of phylogenetic evolution in shaping our "prior bodies" as the key constitutive factor molding how we predict ourselves and other agents (Allen \& Tsakiris, 2019). In advance of any ontogenetic development, one is born with homeostatic and morphological features which shape the expected statistics of one's life, and these in turn provide a rich generative model which can be inverted to understand a wide range of human social behaviors.

Developing this view, we recently proposed a computational model of interoceptive self-inference (Allen, Levy, Parr, \& Friston, 2019). Our model argues that the visceral body provides a fundamental 
constraint on belief precision, and that interoception serves to sample these rhythms so as to better estimate expected uncertainty. This model formalizes other more conceptual accounts of interoceptive inference in the domain of emotion (Barrett \& Simmons, 2015; Chanes \& Barrett, 2016; Seth, 2013; Seth \& Tsakiris, 2018), selfhood (Ainley, Apps, Fotopoulou, \& Tsakiris, 2016; Apps \& Tsakiris, 2014; Limanowski \& Blankenburg, 2013), and metacognition (Petzschner, Weber, Gard, \& Stephan, 2017). Our model generalizes beyond these to argue that the primary homeostatic rhythms of the body fundamentally constrain prior beliefs about the precision, or confidence, of both interoceptive and exteroceptive belief updates. This to say, visceral rhythms embed primary control dynamics, or hyperpriors, on the agent's landscape of precision. This in turn dictates the confidence I assign to any shift in my posterior beliefs and provides a useful starting point for estimating self-precision in others.

\section{Interoceptive Self-Inference B.}

A.

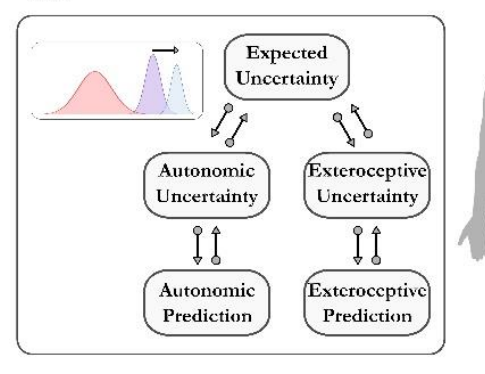

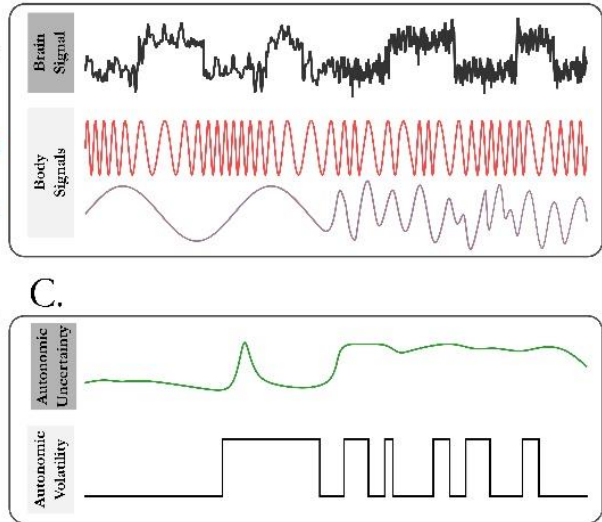

Figure 1. Interoceptive Self-Inference Model. A) Hierarchical precision-weighted inferences integrate confidence signals from the internal and external environment into an overall estimate of expected uncertainty. B) For example, slow respiratory oscillations stabilize cardiac cycles, resulting in low autonomic uncertainty. In contrast, a volatile breath pattern increases baseline neural uncertainty, as illustrated in a simulated brain response to a steady state exteroceptive input. C) These fluctuations can be modeled, e.g., by dynamic reinforcement learning approach in which the volatility of interoceptive state transitions inflates the estimate of autonomic uncertainty. Through inversion of the self-inference model to conspecifics, agents can predict the confidence of others' beliefs.

To illustrate the core of our model (summarized in Figure 1), consider the following example of sensory attenuation in the retina. In the eye, the pulsation of blood across the optic disk at each heartbeat distorts the retinal surface, briefly occluding ascending sensory information. In a hierarchical brain, this predictable fluctuation of precision is a crucial learning signal, which can be sampled via interoception to improve estimates of expected uncertainty (Parr \& Friston, 2017; Pulcu \& Browning, 2019). Expected uncertainty in turn provides an invaluable control signal dictating how much I should update my beliefs in the face of new information; Bayesian decision theory tells us that when confronted with a volatile environment (or a volatile colleague), one should more rapidly update their beliefs in response to prediction error.

Through simulation, we show that this simple coupling of sensory precision to the rhythm of homeostasis enforces a primary interaction between the body and our perception of the world. On our model, lesioning afferent viscero-sensory information caused a cascade of interoceptive prediction error which elicit psychosomatic hallucinations, blunted belief updating, and attenuated physiological reactions. These domain-general alterations in precision ultimately cause agents to update their higherorder beliefs, resulting in top-down metacognitive biases (i.e., mis-estimation of expected uncertainty) that characterize many psychiatric and psycho-social illnesses (Lawson, Mathys, \& Rees, 2017; Powers, 
Mathys, \& Corlett, 2017). In the inverse, maladaptive prior beliefs about self-uncertainty can elicit misperception or hyper-arousal in the interoceptive domain. This equips the model with a deeply circular, enactive causation; my expectation of confidence in the world constrains my visceral inference and regulation, and the statistics of visceral rhythms constrain my exteroceptive percepts and beliefs.

Here perhaps is where there is the most potential for crosstalk between the model of Veissièr and colleagues and interoceptive-self inference. Our model suggests that agents are imbued at birth with a repertoire of "embodied priors" or statistical regularities dictated by their morphological forms, which act as hyper-parameters on meta-cognition and learning (Allen \& Tsakiris, 2019). In particular, these priors influence the confidence or precision dictating the perceptual and emotional salience we assign to various interoceptive and exteroceptive outcomes. The notion of Variational Niche Construction developed by Veissière and others can be cast as building ontogenetic refinement on top of these fundamental constraints (Bruineberg, Kiverstein, \& Rietveld, 2018; Bruineberg, Rietveld, Parr, van Maanen, \& Friston, 2018). That is to say; in thinking through other minds we demarcate novel boundaries of salience, refining the embodied set-points that define a landscape of precision for agents. We maintain however the hegemony of the phylogenetic body in setting these starting points; ultimately, the strongest possible source one can sample from concerning the volatility of others is found within oneself.

\section{Acknowledgements}

MA is supported by a Lundbeckfonden Fellowship (R272-2017-4345), and the AIAS-COFUND II fellowship programme that is supported by the Marie Skłodowska-Curie actions under the European Union's Horizon 2020 (Grant agreement no 754513), and the Aarhus University Research Foundation.

References

Ainley, V., Apps, M. A. J., Fotopoulou, A., \& Tsakiris, M. (2016). 'Bodily precision': A predictive coding account of individual differences in interoceptive accuracy. Philosophical Transactions of the Royal Society B: Biological Sciences, 371(1708), 20160003. https://doi.org/10.1098/rstb.2016.0003

Allen, M., \& Friston, K. J. (2018). From cognitivism to autopoiesis: Towards a computational framework for the embodied mind. Synthese, 195(6), 2459-2482. https://doi.org/10.1007/s11229-016-1288-5

Allen, M., Levy, A., Parr, T., \& Friston, K. J. (2019). In the Body's Eye: The Computational Anatomy of Interoceptive Inference. BioRxiv, 603928. https://doi.org/10.1101/603928

Allen, M., \& Tsakiris, M. (2019). The body as first prior: Interoceptive predictive processing and the primacy. In The Interoceptive Mind: From Homeostasis to Awareness (First Edition, pp. 2745). Great Clarendon Street, Oxford, OX2 6DP: Oxford University Press.

Apps, M. A., \& Tsakiris, M. (2014). The free-energy self: A predictive coding account of selfrecognition. Neuroscience \& Biobehavioral Reviews, 41, 85-97.

Barrett, L. F., \& Simmons, W. K. (2015). Interoceptive predictions in the brain. Nature Reviews Neuroscience, 16(7), 419-429. https://doi.org/10.1038/nrn3950

Bruineberg, J., Kiverstein, J., \& Rietveld, E. (2018). The anticipating brain is not a scientist: The free-energy principle from an ecological-enactive perspective. Synthese, 195(6), 2417-2444. https://doi.org/10.1007/s11229-016-1239-1

Bruineberg, J., Rietveld, E., Parr, T., van Maanen, L., \& Friston, K. J. (2018). Free-energy minimization in joint agent-environment systems: A niche construction perspective. Journal of Theoretical Biology, 455, 161-178. https://doi.org/10.1016/j.jtbi.2018.07.002

Chanes, L., \& Barrett, L. F. (2016). Redefining the Role of Limbic Areas in Cortical Processing. Trends in Cognitive Sciences, 20(2), 96-106. https://doi.org/10.1016/j.tics.2015.11.005

Friston, K., \& Frith, C. (2015). A Duet for one. Consciousness and Cognition, 36, 390-405. https://doi.org/10.1016/j.concog.2014.12.003 
Friston, K. J., \& Frith, C. D. (2015). Active inference, communication and hermeneutics. Cortex, 68, 129-143. https://doi.org/10.1016/j.cortex.2015.03.025

Gallagher, S., \& Allen, M. (2018). Active inference, enactivism and the hermeneutics of social cognition. Synthese, 195(6), 2627-2648. https://doi.org/10.1007/s11229-016-1269-8

Lawson, R. P., Mathys, C., \& Rees, G. (2017). Adults with autism overestimate the volatility of the sensory environment. Nature Neuroscience, 20(9), 1293-1299. https://doi.org/10.1038/nn.4615

Limanowski, J., \& Blankenburg, F. (2013). Minimal self-models and the free energy principle. Frontiers in Human Neuroscience, 7. https://doi.org/10.3389/fnhum.2013.00547

Parr, T., \& Friston, K. J. (2017). Uncertainty, epistemics and active inference. Journal of The Royal Society Interface, 14(136), 20170376. https://doi.org/10.1098/rsif.2017.0376

Petzschner, F. H., Weber, L. A. E., Gard, T., \& Stephan, K. E. (2017). Computational Psychosomatics and Computational Psychiatry: Toward a Joint Framework for Differential Diagnosis. Biological Psychiatry, 82(6), 421-430. https://doi.org/10.1016/j.biopsych.2017.05.012

Powers, A. R., Mathys, C., \& Corlett, P. R. (2017). Pavlovian conditioning-induced hallucinations result from overweighting of perceptual priors. Science, 357(6351), 596-600. https://doi.org/10.1126/science.aan3458

Pulcu, E., \& Browning, M. (2019). The Misestimation of Uncertainty in Affective Disorders. Trends in Cognitive Sciences. https://doi.org/10.1016/j.tics.2019.07.007

Ramstead, M. J. D., Kirchhoff, M. D., Constant, A., \& Friston, K. J. (2019). Multiscale integration: Beyond internalism and externalism. Synthese. https://doi.org/10.1007/s11229-019-02115-x

Ramstead, M. J., Kirchhoff, M. D., \& Friston, K. J. (2019). A tale of two densities: Active inference is enactive inference. Adaptive Behavior, 1059712319862774. https://doi.org/10.1177/1059712319862774

Seth, A. K. (2013). Interoceptive inference, emotion, and the embodied self. Trends in Cognitive Sciences, 17(11), 565-573. https://doi.org/10.1016/j.tics.2013.09.007

Seth, A. K., \& Tsakiris, M. (2018). Being a Beast Machine: The Somatic Basis of Selfhood. Trends in Cognitive Sciences, 22(11), 969-981. https://doi.org/10.1016/j.tics.2018.08.008 